\title{
Ketone bodies and the heart
}

Athanasia K. Papazafiropoulou ${ }^{1}$, Maximos M.Georgopoulos ${ }^{1}$, Nikolaos L. Katsilambros ${ }^{2,3}$

\author{
${ }^{1} 1^{\text {st }}$ Department of Internal Medicine \& Diabetes Center, General Hospital of Piraeus \\ "Tzaneio", Piraeus, Greece \\ ${ }^{2}$ Christeas Hall Research Laboratory, Medical School, National and Kapodistrian \\ University of Athens, Athens, Greece \\ ${ }^{3}$ First Department of Propaedeutic Medicine, National and Kapodistrian University \\ of Athens, "Laiko" University Hospital, Athens, Greece
}

Submitted: 23 July 2021

Accepted: 21 October 2021

Arch Med Sci Atheroscler Dis 2021; 6: e209-e214

DOI: https://doi.org/10.5114/amsad.2021.112475

Copyright (c) 2021 termedia \& Banach

\section{Abstract}

Ketone bodies are low chain organic substances with four carbon atoms, with $\beta$-hydroxybutyric acid and acetone being the main ketone bodies in blood circulation. Under physiological conditions their levels are low while during conditions of oxidative stress, such as exercise, fasting state and acute illness, ketone body levels are increased. Recent findings have shown that in patients with heart failure their plasma concentration is increased. There is a positive correlation between increased energy metabolism of myocardial cells and the levels of $\beta$-hydroxybutyric acid and acetone. Furthermore, it has been hypothesized that the mild ketosis caused by sodium glucose cotransporter 2 inhibitors is one of the possible pathogenetic mechanisms explaining the significant cardiovascular and renal benefits observed in patients with type 2 diabetes treated with these agents. The aim of the present review is to summarize the role of ketone bodies in both normal and pathological conditions, such as heart failure.

Key words: ketone bodies, $\beta$-hydroxybutyric acid, heart failure, diabetes mellitus, ketogenic diet.

\section{Introduction}

Ketone bodies are endogenous metabolites produced by the liver, in particular under conditions of prolonged fasting, insulin deprivation, and extreme exercise [1]. Hepatic ketogenesis produces the three ketone bodies: acetone, acetoacetate (AcAc), and $\beta$-hydroxybutyrate $(\beta \mathrm{OHB})$ acid [1]. Energy metabolism of the healthy heart depends mainly on the presence of free fatty acids and glucose, while ketone bodies and amino acids have a secondary role. Ketone bodies have a key role in energy metabolism of the failing heart. In the presence of heart failure (HF), energy metabolism is dependent on ketone bodies, mainly $\beta \mathrm{OHB}$ acid and acetone $[2,3]$. Ketone bodies are an efficient substrate for cardiac metabolism, as they require less oxygen per molecule of adenosine triphosphate (ATP) generated. Recently, the cardioprotective effects of ketone bodies beyond energetics have been identified [2, 3].

The aim of the present review is to summarize the evidence and discuss the pleiotropic effects of ketone bodies on cardiac metabolism.

\author{
Corresponding author: \\ Athanasia K. \\ Papazafiropoulou MD, MSc \\ $\mathrm{PhD}$ \\ $1^{\text {st }}$ Department \\ of Internal Medicine \\ \& Diabetes Center \\ General Hospital \\ of Piraeus "Tzaneio" \\ 1 Zanni \& Afentouli St \\ 18536 Piraeus, Greece \\ Phone: +30 6979969483 \\ E-mail: \\ pathan@ath.forthnet.gr
}




\section{Ketone bodies' metabolism}

Ketogenesis is the biochemical process of the transformation, through lipolysis, of free fatty acids that are released from adipose tissue, into energy substrates [4]. Readily soluble in blood and water, concentrations of circulating ketone bodies are dynamic and they are oxidized in proportion to their delivery. They are, characteristically, produced in the liver in a fasting state, under a highfat and low-carbohydrate diet (ketogenic diet), during prolonged exercise and at the time of diagnosis of type 1 diabetes (T1D) or during periods of poorly controlled T1D [1]. In the fasting state, fatty acids are mobilized and converted into ketone bodies by the liver, and then are transferred to peripheral tissues, where they undergo oxidation. Moreover, biosynthesis of ketone bodies is related to multiple metabolic pathways, such as the $\beta$-oxidation of fatty acids, the tricarboxylic acid cycle, sterol biosynthesis, de novo lipogenesis, glucose metabolism, and the mitochondrial electron transport chain $[1,4]$. Therefore, the concentration of ketone bodies in the blood is dependent on nutritional and hormonal status.

Ketone bodies can be transported into the circulation without energy cost. They are able to freely pass the blood-brain barrier using the monocarboxylic acid transporters [5]. During conditions of oxidative stress liver ketogenesis is stimulated and high levels of ketone bodies are released into the circulation. Plasma ketone body concentrations in humans range from $<0.1 \mathrm{mM}$ in a fed state up to $6 \mathrm{mM}$ in a prolonged fasted state, and as high as $25 \mathrm{mM}$ in diabetic ketoacidosis [6].

The human liver is able to produce up to $300 \mathrm{~g}$ of ketone bodies per day [7]. In non-fasting conditions, metabolism of ketone bodies provides about $5 \%$ of total energy requirements, an amount that is increased by approximately $20 \%$ under fasting conditions [8]. In the non-fasting state insulin inhibits ketogenesis via inhibition of hormone-sensitive lipase, thus preventing triacylglycerol breakdown to glycerol and fatty acids, and ultimately decreases the substrate for ketogenesis. Con- versely, during a fasted state, adipose tissue lipolysis releases fatty acids for hepatic ketogenesis.

Oxidation of ketone bodies yields more ATP per $g$ than glucose and therefore ketone bodies are classified as "super fuels" $[9,10]$. In particular, the combustion of $100 \mathrm{~g}$ of acetoacetate or $\beta \mathrm{OHB}$ acid results in the production of $9,400 \mathrm{~g}$ and $10,500 \mathrm{~g}$ of ATP, respectively, whereas combustion of $100 \mathrm{~g}$ of glucose results in the production of $8,700 \mathrm{~g}$ of ATP. Ketone bodies are more energetically efficient fuels than other major energy substrates for the heart, particularly fatty acids and glucose [11]. $\beta \mathrm{OHB}$ acid contributes to a $24 \%$ improvement in cardiac efficiency, mitochondrial function, and the stabilization of cellular membrane potential, resulting in enhancement of the antiarrhythmic potential of the myocardial cell $[12,13]$. The above was confirmed in a recent study in mice with hypertrophic hearts, which showed the shift of the energy homeostasis to ketone body consumption [14].

\section{Ketogenic diet}

Ketogenic diets (KD) are very low carbohydrate, high protein, very high fat content diets which induces ketogenesis (Table I). An increasing number of studies are exploring their possible therapeutic effects on many diseases. Intake of carbohydrates with consequent increase in insulin secretion inhibits lipolysis and promotes fat storage [4]. Conversely, the drastic reduction in carbohydrates to levels $\leq 50 \mathrm{~g} /$ day decreases circulating insulin, with the opposite effect. As the KD progresses, a gradual adjustment of metabolism is made to the new energy conditions. After 3-4 weeks the condition is similar to that observed in a fasting state [6].

Application of KD for weight loss dates from 1860 , and was proposed as a treatment of choice in William Osler's manual at the beginning of 1900. In the 1970s, KD became more widely known through the publication of the results of the extreme and not widely accepted Atkins diet [15]. However, over the past 15 years, many randomized studies and reviews have confirmed the

Table I. Macronutrient concentrations of different types of ketogenic diet (\% of total calories)

\begin{tabular}{|lccc|}
\hline Macronutrient substances ratio & Fat (\%) & Protein (\%) & Carbohydrates (\%) \\
\hline Classic KD & 90 & 6 & 4 \\
\hline Modified KD & 87 & 10 & 3 \\
\hline Modified KD & 82 & 12 & 6 \\
\hline Modified KD & 70 & 15 & 15 \\
\hline MCT $(1.9: 1)$ & $* 50 / 21$ & 19 & 10 \\
\hline Low glycemic index $(2: 3)$ & 60 & 28 & 12 \\
\hline Modified Atkins diet & 65 & $29-32$ & $3-6$ \\
\hline
\end{tabular}

KD - ketogenic diet, ${ }^{*} 50 \%$ MCT/21\% LCT, MCT - medium-chain fatty acids, LCT - long-chain fatty acids (Archives of Hellenic Medicine 2019; 36: 611-22). 
relative safety at least in their short-term implementation, but also their good short- and medium-term effectiveness [16]. Based on the research data it appears that KD cause hunger reduction through saturation mechanisms from food proteins, effects on the hormones controlling appetite and direct action by ketone bodies [17].

KD have a favorable impact on the synthesis of saturated fatty acids, an established cardiovascular (CVD) risk factor, as well as on the levels of fasting triglycerides [18, 19]. KD have also been reported to show a significant decrease in blood pressure in overweight subjects [20, 21]. These data indicate that circulating ketone bodies derived from a KD might improve myocardium functioning and can contribute to the treatment of patients with impaired functions of the cardiovascular system $[20,21]$.

$\mathrm{KD}$ are considered to be "the most reliable example of dietary therapy with proven efficacy in certain neurological conditions" [22, 23]. KD and ketone bodies appear to play an important role in improving many neurodegenerative diseases, yet the neuroprotection mechanisms they exercise have not been fully elucidated. In addition to drug resistant epilepsy where a ketogenic diet has been shown to be effective, some reviews and studies also show a potential benefit in motor neuron disease, neurotrauma, brain injury, multiple sclerosis and migraine $[23,24]$.

At this point it must be mentioned that, on the other hand, literature data minimize the enthusiasm for KD. The use of KD for the treatment of pediatric epilepsy has been associated with adverse effects, ranging from fatigue, weakness, and gastrointestinal disturbances, to cardiac arrhythmias $[25,26]$. Low-density lipoprotein cholesterol and apo-B-containing lipoprotein levels may fail to improve, or even significantly increase, with a KD despite weight loss [27]. In patients with T2D, a meta-analysis of randomized long-term studies comparing the KD with low-fat diets for weight loss showed no difference in glycemic control [28]. Finally, studies in animal models $[29,30]$ and children treated with $\mathrm{KD}[31,32]$ have suggested retardation in skeletal development and reduction in bone mineral density.

\section{Supply of ketone bodies to the heart}

Cardiomyocytes are characterized by a high density of mitochondria that makes them capable of oxidizing various substrates to produce ATP [33]. However, whether or not the heart has ketogenic capacity is debatable. In adults, the heart consumes more energy than any other tissue (about $400 \mathrm{kcal} / \mathrm{kg}$ of myocardial tissue/day) [33]. As already mentioned, free fatty acids (in both non-esterified and esterified form) and carbohy- drates (glucose and lactose) are the main sources of energy. It is noted that their oxidation represents about $90 \%$ of cardiac production of ATP. The metabolism of ketone bodies and branched chain amino acids is an alternative energy source for the myocardium $[10,34]$. In particular, under regular feeding conditions, the main energy source of the cardiomyocytes is oxidation of free fatty acids, which provide about $60 \%$ of the requirements in ATP, while the remaining $40 \%$ of myocardial requirements in ATP are provided by glucose oxidation [10, 34]. However, in heart failure, there are increases in circulating levels of ketone bodies, mainly $\beta \mathrm{OHB}$, along with up-regulated capacity of the failing heart to oxidize ketone bodies [10, 34].

Cardiac diseases are associated with loss of metabolic flexibility. Even in early stages of structural heart diseases, substrate utilization switches from fatty acids to glucose utilization, and oxidative metabolism is reduced $[10,34]$. Under hypoxia, the main source of ATP is shifted from $\beta$-oxidation of fatty acids to glucose catabolism as the glycolysis may even function under anaerobic conditions (anaerobic glycolysis). However, as the output of ATP by this pathway is significantly lower than that of mitochondrial oxidative metabolism, more efficient sources of energy are required $[10,34]$.

Separate studies demonstrated that circulating ketone concentrations and cardiac ketone utilization are increased in a variety of clinical conditions, including HF with reduced ejection fraction (HFrEF) and HF with preserved ejection fraction [35-38]. Ketone bodies are a favorable substrate of energy production as their conversion to acetyl coenzyme $A$ is much easier compared to the corresponding conversion of free fatty acids and glucose [10,34]. Plasma concentration of ketone bodies is increased in HF and is positively correlated with ventricular filling pressure [39]. Indeed, a positive correlation was found in patients with HF between the increase in myocardial energy costs and levels of $\beta \mathrm{OHB}$ acid and acetone, leading to the conclusion that ketone bodies could be used as bio-indicators in patients with HF [40]. Data from both human and experimental models confirm the use of ketone bodies as a cardiac energy substrate under ischemic conditions [38, 40].

Increasing circulating ketone concentrations via KD or infusion of $\beta O H B$ ameliorated pathological cardiac remodeling and improved cardiac function in small and large animal models of HF [41, 42]. The importance of the consumption of ketone bodies by the myocardium in HF has been demonstrated in an experimental model of Cre-loxBDH1 $\mathrm{KO}$ mice whose hearts did not have the oxidation capacity of $\beta \mathrm{OHB}$ acid (and not acetoacetate acid) [41]. Mouse BDH1-CO hearts showed increased fasting stress and pathological cardiac reflow af- 
ter pressure overload/ischemic damage (aortic desquamation/myocardial infarction) compared to mouse hearts expressing the $\mathrm{BDH} 1$ gene and able to use $\beta \mathrm{OHB}$ acid. Pathological cardiac remodeling in response to pressure overload was also more pronounced in mice with cardiomyocyte-specific knockout of succinyl-CoA:3-ketoacid-CoA transferase [42]. It has also been shown that ketone bodies improve cardiac function with reduction in pathological cardiac remodeling in animal models of HF, associated with increased markers of myocardial uptake and oxidation of ketones [43]. Plasma $\beta \mathrm{OHB}$ and cardiac utilization are also increased in patients with diabetes and arrhythmogenic cardiomyopathy, suggesting that the ketogenic shift is the cardiac response to stress [44, 45] (Figure 1).

\section{Ketone bodies and diabetic heart}

Diabetic patients suffering from HF have been observed to have an increased cardiac uptake of ketone bodies as compared to those without diabetes [45]. Chronic insulin resistance in the diabetic heart results in alterations of fuel availability and change of the affinity and utilization abilities of myocytes for different substrates [46], with free fatty acids becoming the preferred substrate, which leads to significant reduction of energy efficiency and accumulation of toxic byproducts that exacerbate HF and insulin resistance [47]. Mizuno et al. [45] demonstrated that in diabetic HF the uptake of total ketone bodies is higher than in nondiabetic HF. This suggests that ketone bodies serve as a partial energy source replacement in the human diabetic heart.
Ketosis is a common finding in T1D, especially at the diagnosis of the disease. Therefore, increased plasma concentrations of ketone bodies in patients with T1D entail an increase in tissue availability, including the heart. In addition, in diabetes an increase in free fatty acids is observed as a result of increased lipolysis in fat tissue. Given the competition between these energy substrates [48], it is not entirely clear which dominates cardiac metabolism in the presence of diabetes. There is, however, significant evidence that the consumption of free fatty acids is increasing in the diabetic heart, at the expense of glucose [49] intake, and it is even estimated that the use of free myocardial fatty acids accounts for $>90 \%$ of the ATP requirements by the diabetic myocardium [50]. These result in steatosis and oxidative stress [51] of the diabetic myocardium, which are the main causative factors for diastolic heart dysfunction and ultimately diabetic cardiomyopathy. In contrast, it has been suggested that the increased consumption of ketone bodies from the heart in diabetes represents an adaptive mechanism aimed at better cardiac function.

SGLT2 inhibitors are a new class of antidiabetic agents which, in addition to reducing plasma glucose levels, have significant pleiotropic effects. Inhibition of SGLT2 results in glycosuria, which thereby lowers plasma glucose levels, resulting in a decrease in insulin levels and an increase in the levels of glucagon in the fasting state [44, 45]. The results of large clinical trials have shown favorable effects of SGLT2 inhibitors on cardiovascular and renal outcomes $[52,53]$. One of the

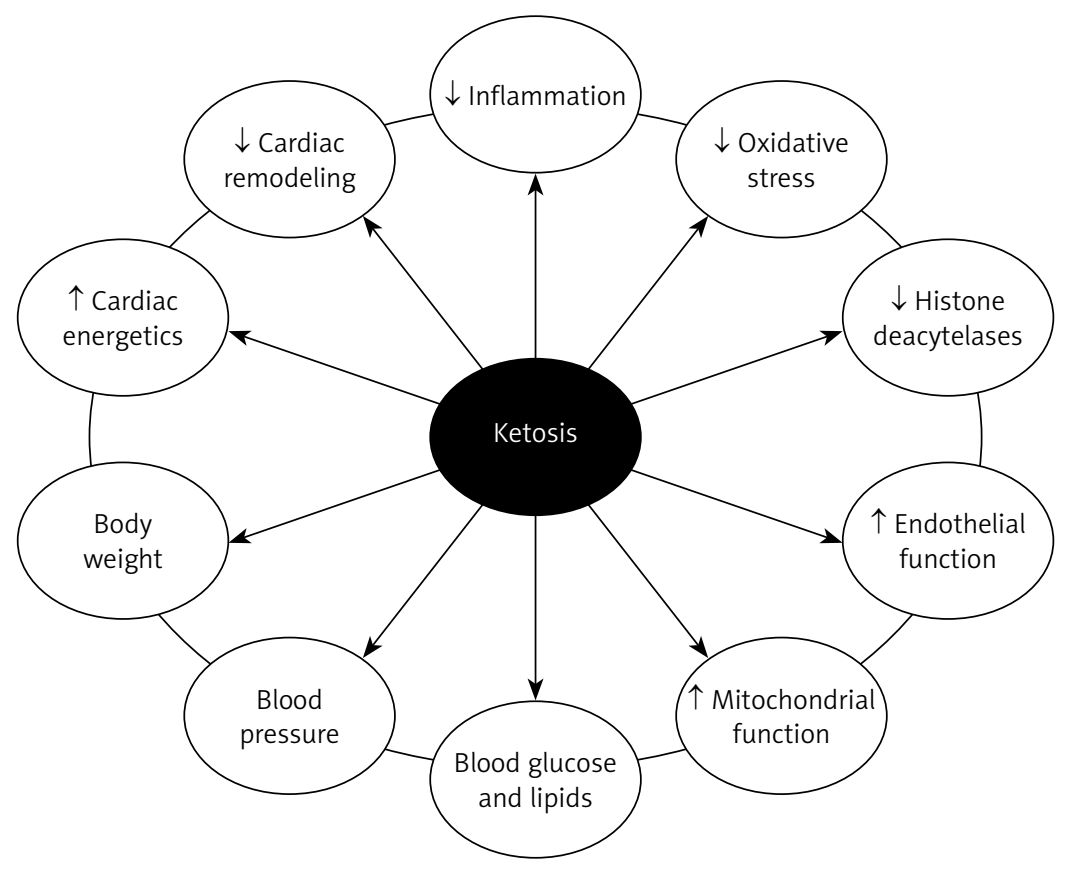

Figure 1. Ketosis 
possible pathogenetic mechanisms explaining these effects is the mild ketosis caused by SGLT2 inhibitor therapy [11]. SGLT2 inhibitors increase the production of ketone bodies in the liver by increasing glucagon levels and decreasing the levels of plasma insulin [11]. Is seems that mild ketosis acts like a fuel for the failing heart and, therefore, therapy with SGLT2 inhibitors in diabetic patients with HF might improve cardiac energetics and cardiac efficiency[11].

\section{Conclusions}

Ketone bodies are not only products of metabolism but they also have a key role in providing energy to vital organs, such as the brain and heart, in both normal and pathological conditions. Only recently, based on the results of large clinical trials with newer antidiabetic agents, the role of ketone bodies in supplying energy in myocardial failure conditions was better understood. Therefore, new prospects are opening with ketone bodies playing a key role in the management of heart diseases as energy substrates.

\section{Conflict of interest}

The authors declare no conflict of interest.

\section{References}

1. Puchalska P, Crawford PA. Multi-dimensional roles of ketone bodies in fuel metabolism, signaling, and therapeutics. Cell Metab 2017; 25: 262-84.

2. Bhanpuri NH, Hallberg SJ, Williams PT, et al. Cardiovascular disease risk factor responses to a type 2 diabetes care model including nutritional ketosis induced by sustained carbohydrate restriction at 1 year: an open label, non-randomized, controlled study. Cardiovasc Diabetol 2018; 17: 56.

3. Gjuladin-Hellon T, Davies IG, Penson P, et al. Effects of carbohydrate restricted diets on low-density lipoprotein cholesterol levels in overweight and obese adults:a systematic review and meta-analysis. Nutr Rev 2019; 77 : 161-80.

4. Randle PJ, Garland PB, Hales CN, et al. The glucose fatty-acid cycle. Its role in insulin sensitivity and the metabolic disturbances of diabetes mellitus. Lancet 1963; 1: 785-9.

5. Kalapos MP. On the mammalian acetone metabolism: From chemistry to clinical implications. Biochim Biophys Acta 2003; 1621: 122-39.

6. Cahill FG Jr. Fuel metabolism in starvation. Annu Rev Nutr 2006; 26: 1-22.

7. Koeslag JH, Noakes TD, Sloan AW. Post-exercise ketosis. J Physiol 1980; 301: 79-90.

8. Owen OE, Morgan AP, Kemp HG, et al. Brain metabolism during fasting. I Clin Investig 1967; 46: 1589-95.

9. Robisnon AM, Williamson DH. Physiological roles of ketone bodies as substrates and signals in mammalian tissues. Physiol Rev 1980; 60: 143-87.

10. Lopaschuk GD, Ussher JR, Folmes CD, et al. Myocardial fatty acid metabolism in health and disease. Physiol Rev 2010; 90: 207-58
11. Ferrannini E, Mark M, Mayoux E. CV protection in the EMPA-REG OUTCOME trial: A "Thrifty Substrate" hypothesis. Diabetes Care 2016; 39: 1108-14.

12. Cotter DG, Schugar RC, Crawford PA. Ketone body metabolism and cardiovascular disease. Am J Physiol Heart Circ Physiol 2013; 304: H1060-76.

13. Shimazu T, Hirschey MD, Newman J, et al. Suppression of oxidative stress by beta-hydroxybutyrate, an endogenous histone deacetylase inhibitor. Science 2013; 339: 211-4.

14. Kalra S, Jain A, Ved J, et al. Sodium-glucose cotransporter 2 inhibition and health benefits: the Robin Hood effect. Indian J Endocrinol Metab 2016; 20: 725-9.

15. Anonymous. A critique of low-carbohydrate ketogenic weight reduction regimens: a review of Dr Atkins' diet revolution. JAMA 1973; 224: 1415-9.

16. Paoli A. Ketogenic diet for obesity: friend or foe? Int J Environ Res Public Health 2014; 11: 2092-307.

17. Johnstone AM, Horgan GW, Murison SD, et al. Effects of a high-protein ketogenic diet on hunger, appetite, and weight loss in obese men feeding ad libitum. Am J Clin Nutr 2008; 87: 44-55.

18. Feinman RD, Pogozelski WK, Astrup A, et al. Dietary carbohydrate restriction as the first approach in diabetes management: critical review and evidence base. Nutrition 2015; 31: 1-13.

19. Volek JS, Phinney SD, Forsythe CE, et al. Carbohydrate restriction has a more favorable impact on the metabolic syndrome than a low-fat diet. Lipids 2009; 44: 297-309.

20. Cicero AF, Benelli $M$, Brancaleoni $M$, et al. Middle and long-term impact of a very low-carbohydrate ketogenic diet on cardiometabolic factors: a multi-center, cross-sectional, clinical study. High Blood Press Cardiovasc Prev 2015; 22: 389-94.

21. Mayer SB, Jeffreys AS, Olsen MK, et al. Two diets with different haemoglobin A1c and antiglycaemic medication effects despite similar weight loss in type 2 diabetes. Diabetes Obes Metab 2014; 16: 90-3.

22. Statfstrom CE, Rho JM. The ketogenic diet as a treatment paradigm for diverse neurological disorders. Front Pharmacol 2012; 3: 59.

23. Vlachos D, Katsilambros N. Ketogenic diets: history, physiology, current perspectives. Arch Hellen Med 2019; 36: 611-22.

24. McDonald TJW, Cervenka MC. Ketogenic diets for adult neurological disorders. Neurotherapeutics 2018; 15: 1018-31.

25. Kang HC, Chung DE, Kim DW, Kim HD. Early- and late-onset complications of the ketogenic diet for intractable epilepsy. Epilepsia 2004; 45: 1116-23.

26. Kwiterovich PO Jr, Vining EP, Pyzik P, Skolasky R Jr, Freeman JM. Effect of a high-fat ketogenic diet on plasma levels of lipids, lipoproteins, and apolipoproteins in children. JAMA 2003; 290: 912-20.

27. Retterstøl K, Svendsen M, Narverud I, Holven KB. Effect of low carbohydrate high fat diet on LDL cholesterol and gene expression in normal-weight, young adults: a randomized controlled study. Atherosclerosis 2018; 279: 52-61.

28. Bueno NB, de Melo IS, de Oliveira SL, da Rocha Ataide T. Very-low-carbohydrate ketogenic diet v. low-fat diet for long-term weight loss: a meta-analysis of randomised controlled trials. Br J Nutr 2013; 110: 1178-87.

29. Bielohuby M, Matsuura M, Herbach N, et al. Short-term exposure to low-carbohydrate, high-fat diets induces low bone mineral density and reduces bone formation in rats. J Bone Miner Res 2010; 25: 275-84. 
30. Wu X, Huang Z, Wang X, et al. Ketogenic diet compromises both cancellous and corticol bone mass in mice. Calcif Tissue Int 2017; 101: 412-21.

31. Simm PJ, Bicknell-Royle J, Lawrie J, et al. The effect of the ketogenic diet on the developing skeleton. Epilepsy Res 2017; 136: 62-6.

32. Willi SM, Oexmann MJ, Wright NM, Collop NA, Key LL Jr. The effects of a high-protein, low-fat, ketogenic diet on adolescents with morbid obesity: body composition, blood chemistries and sleep abnormalities. Pediatric 1998; 101: 61-7.

33. Sack MN, Rader TA, Park S, et al. Fatty acid oxidation enzyme gene expression is downregulated in the failing heart. Circulation 1996; 94: 2837-42.

34. Neubauer S, The failing heart - an engine out of fuel. N Engl J Med 2007; 356: 1140-51.

35. Bedi KC, Snyder NW, Brandimarto J, et al. Evidence for intramyocardial disruption of lipid metabolism and increased myocardial ketone utilization in advanced human heart failure. Circulation 2016; 133: 706-16.

36. Aubert G, Martin OJ, Horton JL, et al. The failing heart relies on ketone bodies as a fuel. Circulation 2016; 133: 698-705.

37. Lommi MDJ. Blood ketone bodies in congestive heart failure. J Am Coll Cardiol 1996; 28: 665-72.

38. Voros G, Ector J, Garweg C, et al. Increased cardiac uptake of ketone bodies and free fatty acids in human heart failure and hypertrophic left ventricular remodeling. Circ Heart Fail 2018; 11: e004953.

39. Kupari M, Lommi J, Ventila $M$, et al. Breath acetone in congestive heart failure. Am J Cardiol 1995; 76: 1076-8.

40. Du Z, Shen A, Huang Y, et al. 1H-NMR-based metabolic analysis of human serum reveals novel markers of myocardial energy expenditure in heart failure patients. PLoS One 2014; 9: e88102.

41. Horton JL, Davidson MT, Kurishima C, et al. The failing heart utilizes 3-hydroxybutyrate as a metabolic stress defense. JCl insight 2019; 4: 21.

42. Schugar RC, Moll AR, André d'Avignon D, et al. Cardiomyocyte-specific deficiency of ketone body metabolism promotes accelerated pathological remodeling. Mol Metab 2014; 3: 754-69.

43. Yurista SR, Matsuura TR, Silljé HHW, et al. Ketone ester treatment improves cardiac function and reduces pathologic remodeling in preclinical models of heart failure. Circ Hear Fail 2021; 14: e007684.

44. Song JP, Chen L, Chen X, et al. Elevated plasma b-hydroxybutyrate predicts adverse outcomes and disease progression in patients with arrhythmogenic cardiomyopathy. Sci Transl Med 2020; 12: e8329.

45. Mizuno Y, Harada E, Nakagawa $\mathrm{H}$, et al. The diabetic heart utilizes ketone bodies as an energy source. Metabolism 2017; 77: 65-72.

46. Dei Cas A, Fonarow GC, Gheorghiade M, et al. Concomitant diabetes mellitus and heart failure. Curr Probl Cardiol 2015; 40: 7-43.

47. Qian N, Wang Y. Ketone body metabolism in diabetic and non-diabetic heart failure. Heart Fail Rev 2020; 25 : 817-22.

48. Ferrannini E, Barrett EJ, Bevilacqua S, et al. Effect of fatty acids on glucose production and utilization in man. J Clin Invest 1983; 72: 1737-47.

49. Rodrigues B, Cam MC, McNeill JH. Myocardial substrate metabolism: implications for diabetic cardiomyopathy. J Mol Cell Cardiol 1995; 27: 169-79.
50. Jia G, Whaley-Connell A, Sowers JR. Diabetic cardiomyopathy: a hyperglycaemia-and insulin-resistance-induced heart disease. Diabetologia 2018; 61: 21-8.

51. Levelt E, Mahmod M, Piechnik SK, et al. Relationship between left ventricular structural and metabolic remodeling in type 2 diabetes. Diabetes 2016; 65: 44-52.

52. Zinman B, Wanner C, Lachin JM, et al. Investigators, empagliflozin, cardiovascular outcomes, and mortality in type 2 diabetes, N Engl J Med 2015; 373: 2117-28.

53. Jarcho JA. More evidence for SGLT2 inhibitors in heart failure. N Engl J Med 2020; 383: 1481-2. 